\title{
Cloning and characterization of the pepper CaPAO gene for defense responses to salt-induced leaf senescence
}

\author{
Huai-Juan Xiao ${ }^{1,2}$, Ke-Ke Liu'², Da-Wei Li ${ }^{1}$, Mohamed Hamed Arisha ${ }^{1,3}$, Wei-Guo Chai ${ }^{4}$ and Zhen-Hui Gong ${ }^{1 *}$
}

\begin{abstract}
Background: Pheophorbide a oxygenase (PAO) is an important enzyme in the chlorophyll catabolism pathway and is involved in leaf senescence. It opens the porphyrin macrocycle of pheophorbide $a$ and finally forms the primary fluorescent chlorophyll catabolite. Previous studies have demonstrated the function of PAO during cell death. However, the characterizaton of PAO during leaf senescence induced by environmental factors is not well understood.

Methods: Homology-based cloning and RACE techniques were used to obtain the full-length CDNA of the CaPAO gene. CaPAO expression was determined by quantitative real-time PCR. Function of CaPAO gene were studied using virus-induced gene silencing and transgenic techniques with tobacco plants (Nicotiana tabacum).

Results: A novel PAO gene CaPAO was isolated from pepper (Capsicum annuum L.). The full-length CaPAO cDNA is comprised of $1838 \mathrm{bp}$, containing an open reading frame of $1614 \mathrm{bp}$, and encodes a 537 amino acid protein. This deduced protein belongs to the Rieske-type iron-sulfur superfamily, containing a conserved Rieske cluster. CaPAO expression, as determined by quantitative real-time PCR, was higher in leaves than roots, stems and flowers. It was upregulated by abscisic acid, methyl jasmonate and salicylic acid. Moreover, CaPAO was significantly induced by high salinity and osmotic stress treatments and also was regulated by Phytophthora capsici. The virus-induced gene silencing technique was used to silence the CaPAO gene in pepper plants. After 3 days of high salt treatment, the chlorophyll breakdown of CaPAO-silenced pepper plants was retarded. RD29A promoter-inducible expression vector was constructed and transferred into tobacco plant. After 7 days of salt treatment, the leaves of transgenic plants were severely turned into yellow, the lower leaves showed necrotic symptom and chlorophyll content was significantly lower than that in the control plants.
\end{abstract}

Conclusions: The expression of CAPAO gene was induced in natural senescence and various stresses. The CaPAO gene may be related to defense responses to various stresses and play an important role in salt-induced leaf senescence.

Keywords: Capsicum annuum L, CaPAO gene, Expression analysis, Functional analysis, Tobacco

\section{Background}

Leaf senescence is the final stage of leaf development, ultimately leading to the death of the entire leaf. Although governed by the developmental age, it also can be stimulated by diverse environmental factors, including plant hormones, drought, salinity, extreme temperature, darkness, wounding and pathogenic infection $[1,2]$. Premature leaf senescence can eventually affect the yield of plants under adverse environmental conditions. Therefore, studying leaf senescence will not only strengthen

\footnotetext{
* Correspondence: zhgong@nwsuaf.edu.cn

${ }^{1}$ College of Horticulture, Northwest A\&F University, Yangling, Shaanxi, P. R. China

Full list of author information is available at the end of the article
}

our comprehension of a basic biological process, it also may provide methods to delay plant aging in order to improve agricultural traits of vegetable crops. Pepper (Capsicum annuum L.) is an important vegetable crop that is extensive cultivated worldwide. In recent years, premature senescence of pepper plants caused by various environmental stresses has become a universal phenomenon and considered an important field of research.

Loss of green color, induced by degradation of chlorophyll, is the most visible symptom of leaf senescence. Pheophorbide $a$ oxygenase (PAO) has been regarded as a crucial enzyme in chlorophyll degradation [3, 4]. It oxygenolytically cleaves the porphyrin macrocycle of 
pheophorbide (pheide) $a$ and finally forms the primary fluorescent chlorophyll catabolite (FCC). The $P A O$ gene was initially obtained from maize (designated $Z m L l s 1$ ) and then discovered in other higher plants, such as rice, wheat, tomato, soybean and canola [5-9]. Previous studies showed that the expression of $P A O$ is induced by natural senescence and environmental stresses in plants [10-12]. In Arabidopsis, AtPAO is encoded by the accelerated cell death 1 (ACD1) gene and is homologous to lethal leaf spot 1 (LLS1) of maize. It belongs to a small family of Rieske-type iron-sulfur oxygenases [13]. The absence of $A C D 1$ has been shown to result in the accumulation of PAO and light-independent cell death when senescence is induced in permanent darkness $[14,15]$.

Tobacco (Nicotiana tabacum) is one of the most important model plants for transgenic research. In previous studies, many new genes from other plants have been transferred into tobacco for further research on their functions [16, 17]. The RD29A promoter from Arabidopsis thaliana is a stress-inducible promoter. This promoter contains two dehydration- responsive elements (DREs), which are involved in response to salt, dehydration and low temperature [18]. Using the RD29A promoter instead of the constitutive 35S CaMV promoter for certain genes overexpression minimizes the negative effects on plant growth, which have been widely used in genetic transformation [16].

In recent years, the research on PAO focused on the functional analysis for inhibiting cell death [11, 13, 14]. However, little is known about CaPAO regulatory role after induction expression of inducible-promoter in pepper. This study was conducted to clone this gene from the pepper plant and analyze its molecular characteristics. Patterns of CaPAO gene expression in specific tissues and in response to various stresses were analyzed by quantitative real-time PCR. Furthermore, virus-induced gene silencing (VIGS) and transgenic technologies were used to study CaPAO gene function. The results suggest that $\mathrm{CaPAO}$ may play an important role during leaf senescence and chlorophyll degradation.

\section{Results}

\section{Cloning and Sequence Analysis of CaPAO}

The full-length cDNA designated $C a P A O$ was obtained using in silico cloning and RACE techniques. The transcript consists of 1838 nucleotides, including a 5'-untranslated region (UTR) of $32 \mathrm{bp}$, an ORF of $1614 \mathrm{bp}$ and a 3'-UTR of $192 \mathrm{bp}$ (GenBank accession number KC176709) (Fig. 1). $\mathrm{CaPAO}$ was predicted to encode a 537 amino acid protein with a theoretical molecular weight (MW) of $60.8 \mathrm{kDa}$ and calculated isoelectric point $(\mathrm{p} I)$ of 6.89 . Structural analysis revealed that CaPAO belongs to a Rieske-type iron-sulfur superfamily, containing a conserved Rieske cluster, a mononuclear iron-binding site and a redox-active $\mathrm{CxxC}$ motif in the C-terminal end, which are necessary for oxygen activation (Fig. 1). Subcellular analysis localized CaPAO in the chloroplasts. Further sequence analysis indicated that the deduced $C a P A O$ protein contains a chloroplast transit peptide of 50 amino acid residues with a cleavage site located between $R_{50}$ and $V_{51}$, but it does not contain a signal peptide region or transmembrane helix.

The deduced $\mathrm{CaPAO}$ amino acid sequence showed high homology to other plant PAO sequences via multiple alignments using DNAMAN software (Fig. 1). The percent identities of CaPAO relative to Solanum lycopersicum lethal leaf spot 1-like protein (NP_001234535), N. tabacum PAO1 (ABY19384.1) and PAO2 (ABY19385.1), Vitis vinifera PAO (ACO56118.1), Ricinus communis PAO (XP_002523735.1), Brassica napus PAO (ABD60317.1) and A. thaliana PAO (AEE77964.1), were 90, 85, 88, 76, 75, 71 and $71 \%$, respectively.

A phylogenetic tree, constructed using MEGA5.05 software, was used to investigate the evolutionary relationship of the CaPAO amino acid sequence with PAO proteins of other plants. Two groups were formed using the $14 \mathrm{PAO}$ protein sequences from $C$. annuum L., $N$. tabacum, $S$. lycopersicum, $R$. communis, $V$. vinifera, B. napus, $A$. thaliana, Medicago truncatula, Aegilops tauschi, Pisum sativum and Brassica rapa var. parachinensis (Fig. 2). CaPAO clustered in the first group, which included SILLS1, NtPAO, BoPAO, BrPAO and AtPAO. CaPAO was more closely related to SILLS1, NtPAO than PAO proteins of other plants. All of the above-mentioned bioinformatic analyses demonstrated that $\mathrm{CaPAO}$ should function as a PAO.

\section{Tissue-Specific Expression of CaPAO}

In order to investigate the expression levels of the CaPAO gene in different tissues, total RNA was extracted from the roots, stems, leaves and flowers, and quantitative realtime PCR (qRT-PCR) was performed (Fig. 3a). CaPAO transcripts were detected in all of these tissues and found to be higher in leaves than in other tissues. For different leaf developmental stages, a low level of CaPAO transcript was detected in young and fully mature leaves, but $\mathrm{CaPAO}$ expression was increased in senescent leaves (Fig. 3b).

Induction of signaling molecules, abiotic and biotic stresses In order to study its function in pepper plants, the expression pattern of CaPAO was first analyzed. B12 cultivar seedlings at the six-leaf stage were treated with various stresses, including phytohormones, salt, osmosis and Phytophthora capsici infection, and analyzed by qRT-PCR.

To analyze the response of the CaPAO gene to abiotic stresses, pepper plants were exposed to $400 \mathrm{mM}$ sodium chloride $(\mathrm{NaCl})$ and $400 \mathrm{mM}$ mannitol, and then the abundance of $\mathrm{CaPAO}$ transcripts was analyzed by quantitative RT-PCR. As shown in Fig. 4a, the CaPAO expression 


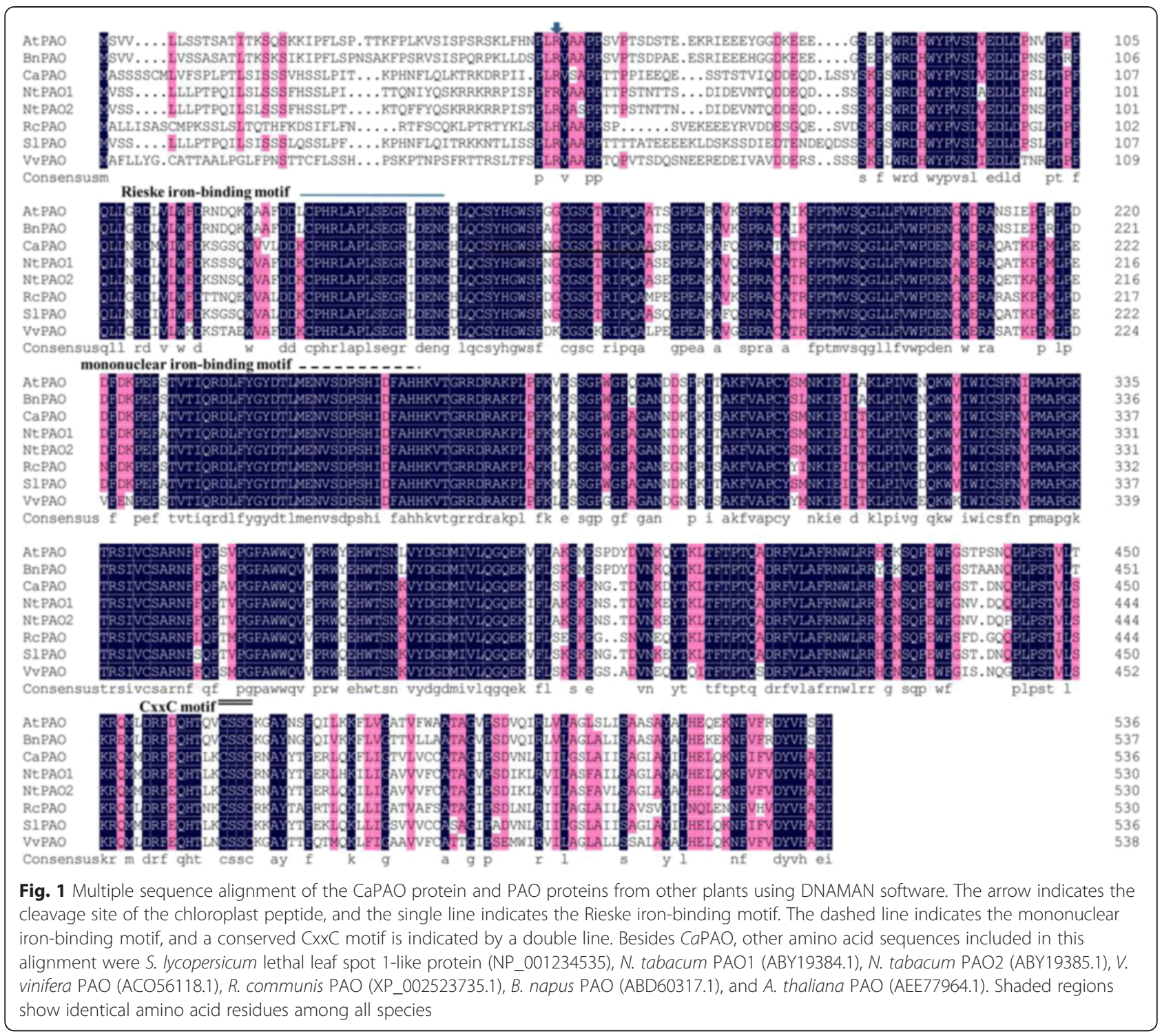

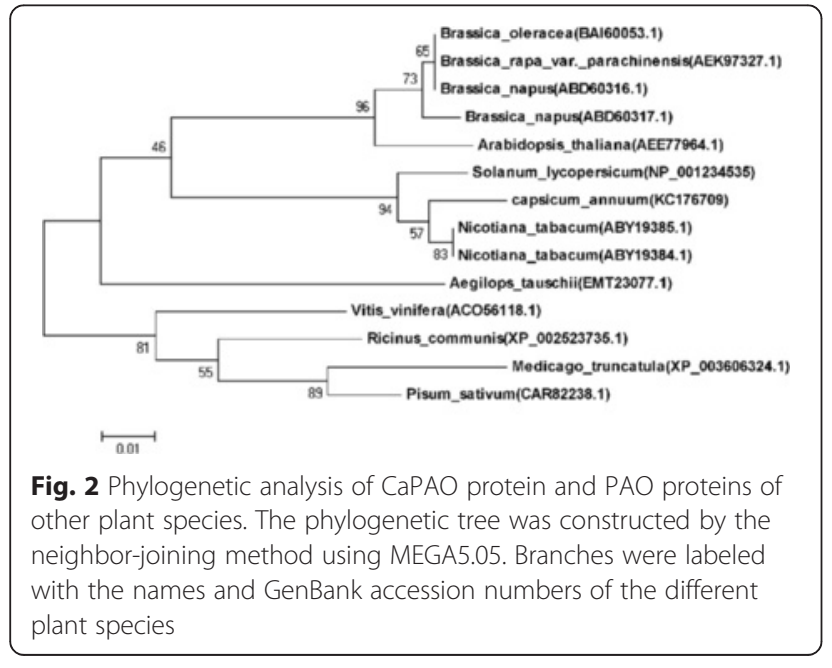

level in plants treated with $400 \mathrm{mM} \mathrm{NaCl}$ began to increase gradually at $4 \mathrm{~h}$ and peaked (11.9-fold) at $24 \mathrm{~h}$, compared to the control $(0 \mathrm{~h})$. With osmotic treatment, CaPAO was induced quickly in pepper plants (Fig. 4b) with a 3.4-fold increase in expression at $2 \mathrm{~h}$ and a stronger 10.7 -fold elevation at $12 \mathrm{~h}$. The highest transcript level was detected at $24 \mathrm{~h}$ (18.7-fold). These results indicate that the increased abundance of the CaPAO gene transcript may be part of the response to abiotic stresses, including high salinity and osmotic stress.

To examine whether stress-related signaling molecules can induce $\mathrm{CaPAO}$ expression, three phytohormones were used to treat pepper leaves. As shown in Fig. 4c, the expression levels of $\mathrm{CaPAO}$ in leaves sprayed with abscisic acid (ABA), methyl jasmonate (MeJA) and salicylic acid (SA) were elevated to different extents. ABA could induce quickly the expression of the $\mathrm{CaPAO}$ gene within the first 

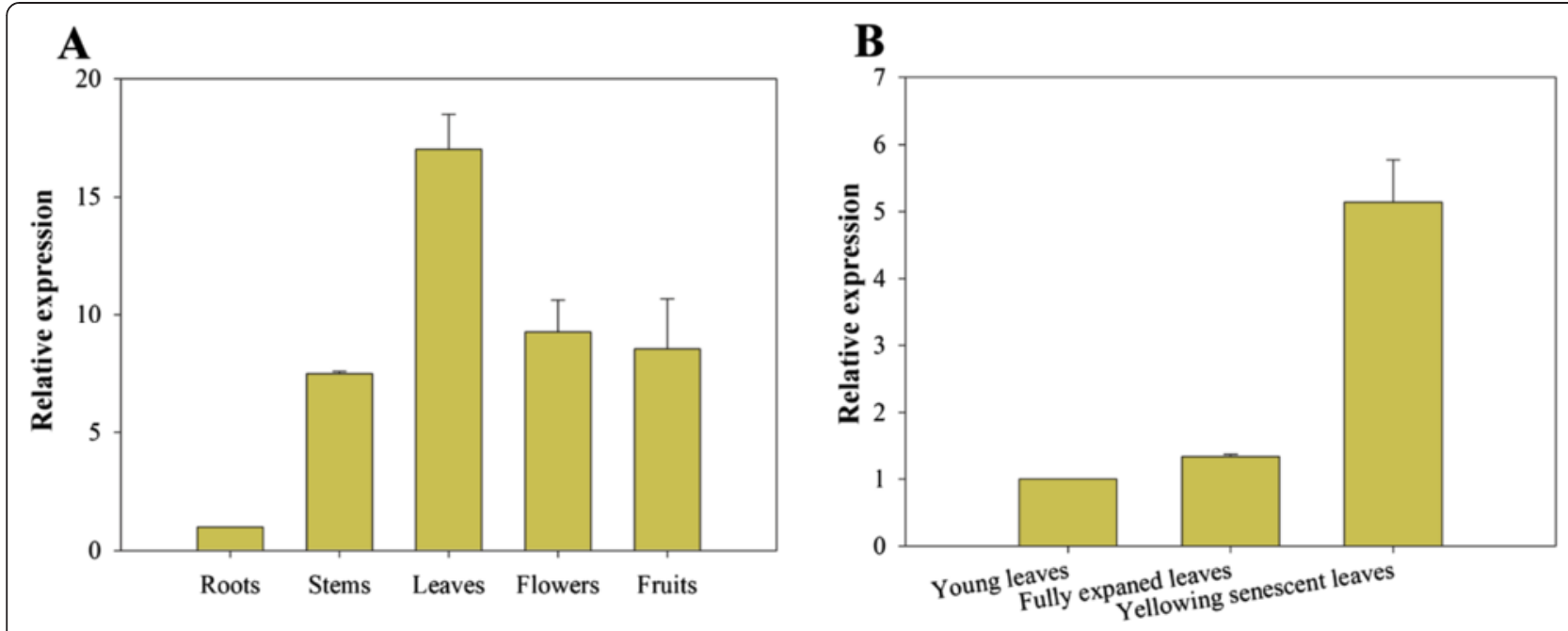

Fig. 3 Tissue-specific expression of CaPAO in pepper. a Tissue-specific expression of CaPAO in pepper roots, leaves, stems and flowers. Relative expression levels of the CAPAO transcript were determined in different tissues in comparison to that in roots; $\mathbf{b}$ The expression profiles of CaPAO during pepper leaf developmental stage. RNA was extracted from young leaves, fully expanded leaves and senescent leaves, respectively. Error bars represent SD for three independent replicates

$4 \mathrm{~h}$ after treatment. Compared to the control, CaPAO transcripts were detected at the highest level (13.8-fold) at $4 \mathrm{~h}$ and then decreased gradually after $8 \mathrm{~h}$. Interestingly, there was a slight up-regulation at $24 \mathrm{~h}$. Spraying pepper plants with MeJA caused a gradual upregulation of CaPAO within the first $4 \mathrm{~h}$, but then it declined slowly. However, the CaPAO expression peaked by $24 \mathrm{~h}$ at a 10.1fold greater level than the control. By contrast, the treatment of SA quickly induced the CaPAO transcript abundance at $2 \mathrm{~h}$ to 7.6 -fold higher than the control and maintained a relatively steady level from $4 \mathrm{~h}$ to $12 \mathrm{~h}$ before a sharp downregulation at $24 \mathrm{~h}$. At $48 \mathrm{~h}$, the CaPAO transcript decreased to nearly the same levels among all of the plants treated with the three phytohormones. These results indicated that the CaPAO gene could be induced and upregulated by all three stress-related signaling molecules tested (ABA, MeJA and SA).

CaPAO expression was enhanced in pepper plants infected with $P$. capsici as shown in Fig. 4 d. The CaPAO transcriptional level was slightly decreased at $3 \mathrm{~h}$ and remained at a steady level from $6 \mathrm{~h}$ to $24 \mathrm{~h}$, compared with the mock control. This transcript then increased rapidly and peaked (12.8-fold) at $48 \mathrm{~h}$. Subsequently, the CaPAO expression level sharply downregulated at $72 \mathrm{~h}$ (5.3-fold) and decreased to 4.9 -fold at $96 \mathrm{~h}$, which was the lowest level after infection relative to the control. These results revealed that $C a P A O$ may be involved in the pepper defense response against pathogens.

\section{VIGS assay of CaPAO Gene in pepper plants Silencing efficiency of CaPAO gene}

The results showed that CaPAO participates in the chlorophyll degradation pathway and is involved in the response to various stresses in pepper plants. To further examine the function of CaPAO in pepper, a tobacco rattle virus (TRV)based VIGS technique was used. Pepper plants inoculated with Agrobacterium for 6-7 weeks were used for the following treatments. An empty vector was applied to plants (TRV2:00) as a negative control. TRV2: CaPDS plants, in the endogenous phytoene desaturase (PDS) gene was silenced to cause photobleaching, were used as positive controls for testing VIGS efficiency. Three weeks after the Agrobacterium inoculation, we found that most of the plants clearly showed symptoms of viral infection. Furthermore, the TRV2: CaPDS plants began to exhibit the photobleached phenotype. These results indicated that VIGS was successfully applied in this experiment. As shown in Fig. 5a, no morphological distinction was observed between the CaPAO-silenced plants (TRV2: $\mathrm{CaPAO}$ ) and the empty vector treated control plants (TRV2:00) $45 \mathrm{~d}$ after inoculation. Simultaneously, $\mathrm{CaPAO}$ transcript levels in empty vector control plants (TRV2:00) and CaPAO-silenced plants (TRV2: $\mathrm{CaPAO}$ ) were examined by quantitative RT-PCR to screen the efficiency of $C a P A O$ gene silencing by VIGS (Fig. 5b). The results showed that the CaPAO transcriptional level was reduced remarkably in $\mathrm{CaPAO}$-silenced plants compared to the empty vector control, which demonstrated that the gene silencing was successful.

\section{Silencing of CaPAO delay chlorophyll breakdown under high salt treatment}

In order to analyse whether the $\mathrm{CaPAO}$ response to salt stress, the leaf discs from empty vector control-treated (TRV2:00) and CaPAO-silenced (TRV2: CaPAO) plants were exposed to various concentrations $(0,300,400$ and $500 \mathrm{mM}$ ) of $\mathrm{NaCl}$ solution with continuous lighting for $3 \mathrm{~d}$. 


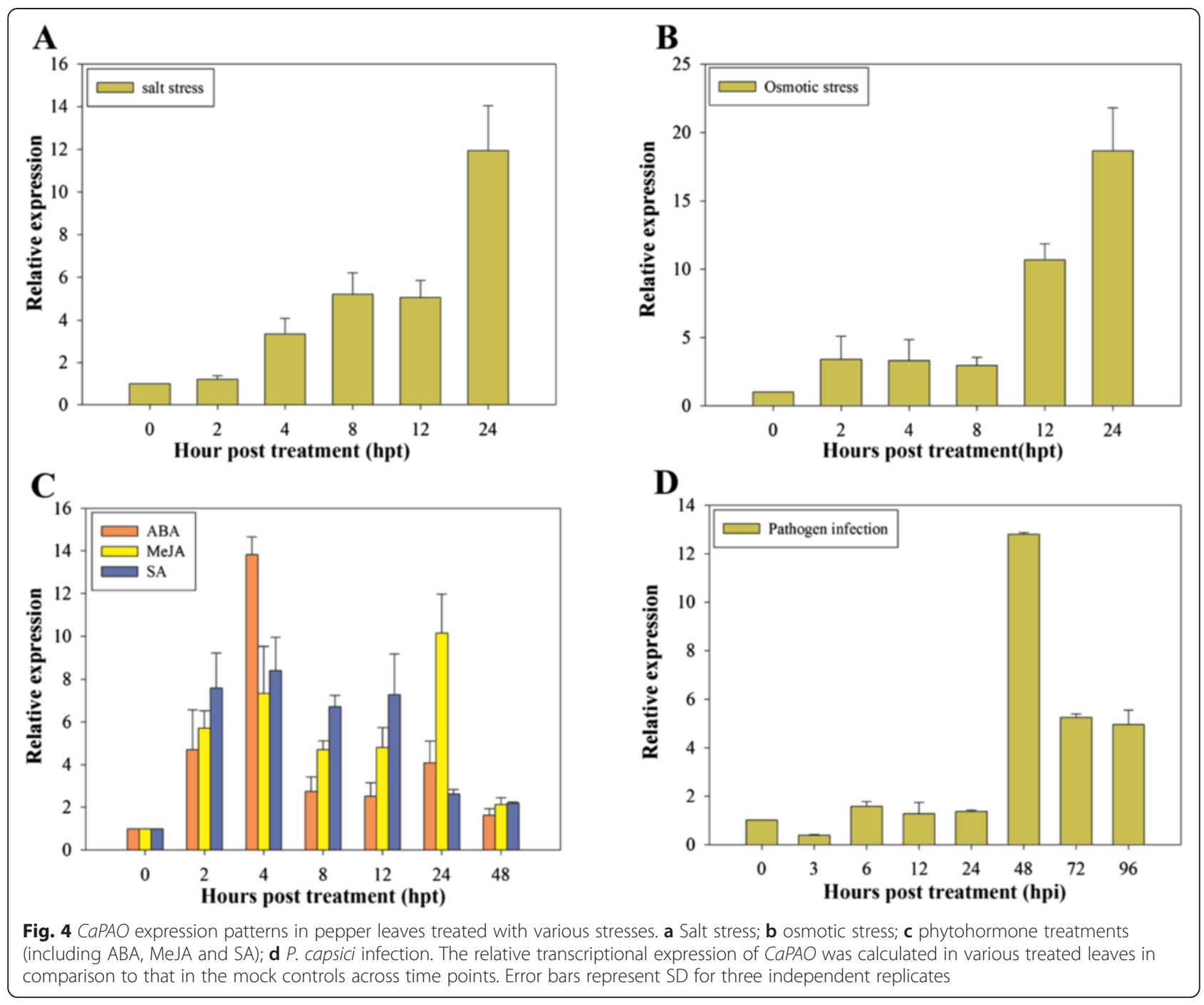

As shown in Fig. 6a, the color of the leaf discs of TRV2:00 plants turned yellow under $300 \mathrm{mM} \mathrm{NaCl}$ treament and the leaf discs appeared to be a bleached phenotype under treatment with higher $\mathrm{NaCl}$ concentrations (400 and $500 \mathrm{mM}$ ). However, the leaf discs of TRV2: CaPAO plants slightly shrinked and their color showed little change. Furthermore, chlorophyll breakdown in TRV2:00 leaves was observed to occur faster than that in TRV2: CaPAO leaves $3 \mathrm{~d}$ after treatment with high $\mathrm{NaCl}$ concentrations (400 and $500 \mathrm{mM}$ ) (Fig. 6b). These results suggested that silencing the CaPAO gene can inhibit chlorophyll breakdown during salt stress-induced leaf senescence in pepper plants.

\section{Effect of RD29A promoter-inducible overexpression of CaPAO on salt tolerance of tobacco Identification of transgenic plants}

To examine the relative contribution of CaPAO to salt stress-induced leaf senescence, tobacco plants were transformed with PVBG2307-PRD29A-CaPAO vector. A sequence of $491 \mathrm{bp}$, which contained a 284-bp fragment of the RD29A promoter and a 207-bp fragment of the CaPAO gene, was produced from genomic DNAs of the positive transgenic $T_{0}$ lines. Nine plants were confirmed to be positive transgenic $T_{0}$ lines. $T_{1}$ and $T_{2}$ generation of transgenic tobacco plants were selected using $50 \mathrm{mg} \cdot \mathrm{L}^{-1}$ kanamycin, and finally obtained transgenic homozygous $\mathrm{T}_{2}$ lines. One $\mathrm{T}_{2}$ positive transgenic plants were used in subsequent studies.

\section{Semi-quantitative RT-PCR analysis of the transgenic tobacco plants after salt stress treatment}

As shown in Fig. 7, CaPAO showed no expression in the CaPAO transgenic tobacco and wild type (WT) plants before salt stress treatment. CaPAO was strongly induced in the leaves of the transgenic plants at $24 \mathrm{~h}$ after treatment with $150 \mathrm{mM} \mathrm{NaCl}$ solution, while it was not expressed in WT plants at all. This may suggest that the CaPAO gene, controlled under the stress-inducible promoter RD29A, was induced to express under exogenous salt treatment in positive transgenic tobacco plants. 

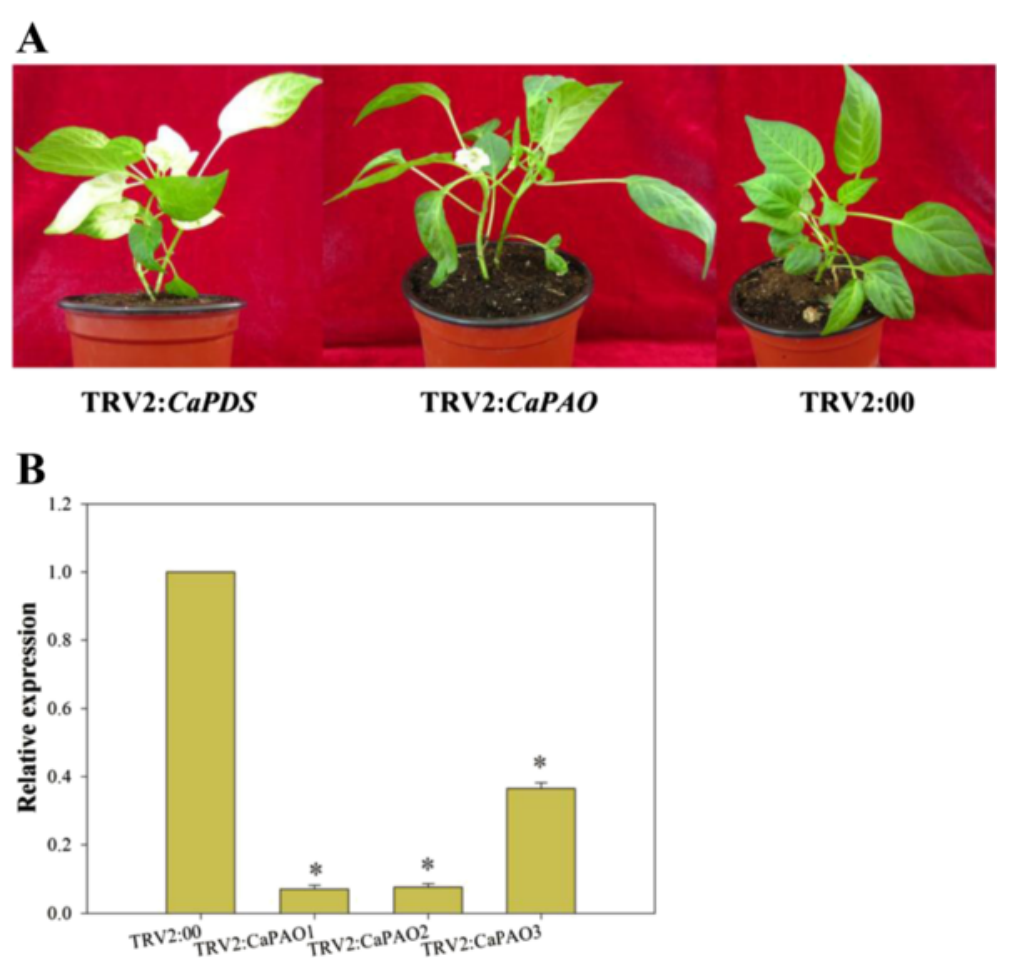

Fig. 5 Efficiency of CAPAO gene-silencing in pepper plants. a Phenotypes of gene-silenced pepper plants $45 \mathrm{~d}$ after inoculation. Left: control plant (TRV2:00); middle: CaPDS-silenced plant (TRV2: CaPDS); right: CaPAO-silenced plant (TRV2: CaPAO). b Quantitative real time-PCR analysis of CaPAO expression levels in leaves of CaPAO-silenced plants (TRV2: CaPAO1, TRV2: CaPAO2 and TRV2: CaPAO3) and control plants (TRV2:00) 45 d after inoculation. Error bars represent SD for three independent replicates. Asterisks indicate a significant difference $(p<0.05)$ compared to TRV2:00 leaves

Phenotype of transgenic tobacco plants after salt stress treatment Before salt stress treatment, there were no phenotypic differences between CaPAO transgenic and WT plants (Fig. 8a). At early stage of the salt stress treatment (150 mM NaCl solution), both leaves of transgenic and WT plants wilted with more serious wilting degree in the WT plants. Along with the time of treatment, the lower part of leaves became yellow. After salt treatment for $7 \mathrm{~d}$, the leaves of the transgenic plants became yellow and the edge of the lower leaves showed necrotic symptoms after salt stress treatment, while the upper young leaves were green. However, leaves of WT plants appeared to shrink, with

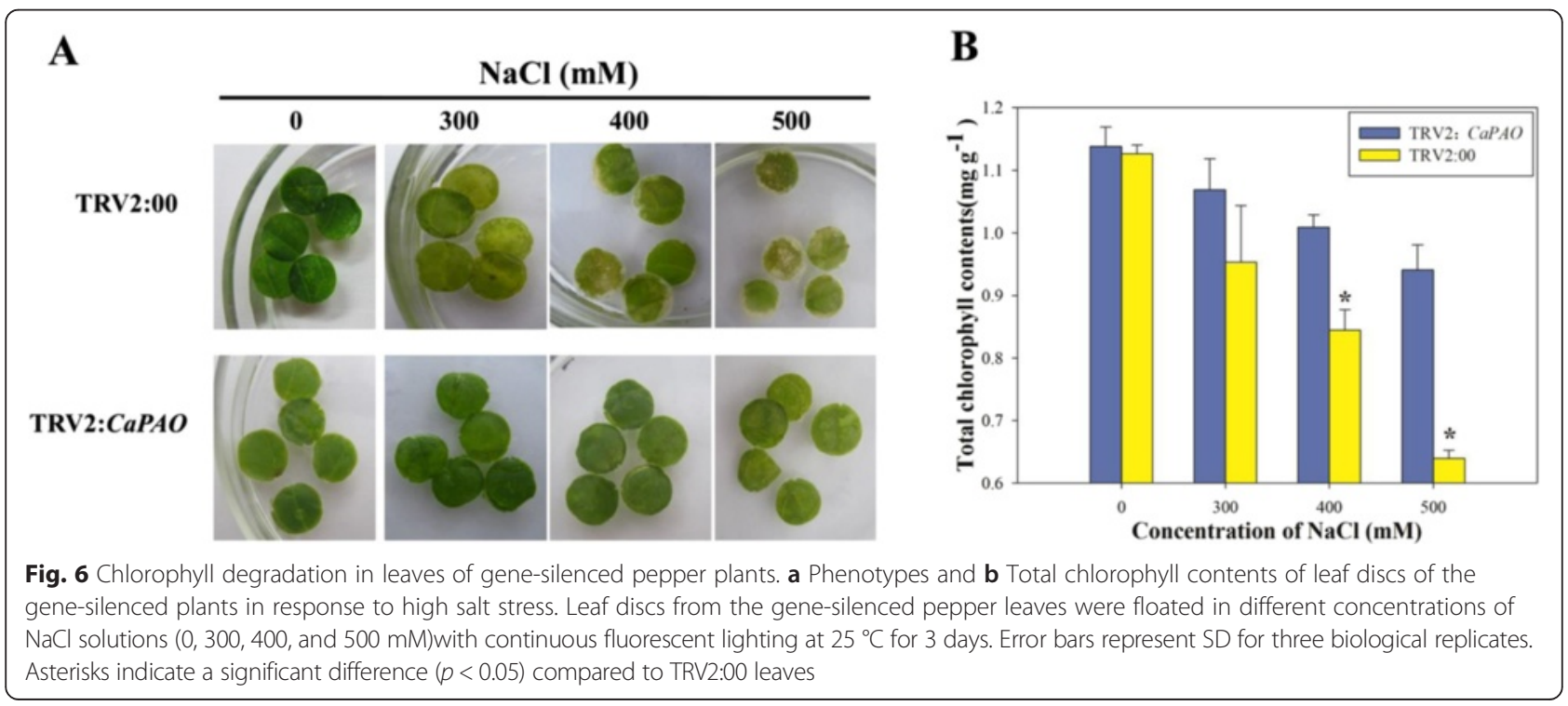




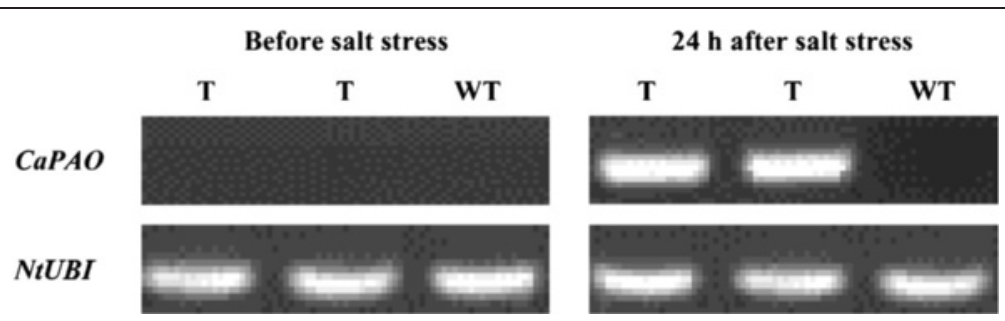

Fig. 7 RT-PCR analysis of CaPAO in transgenic tobacco plants with and without salt treatment under $150 \mathrm{mM} \mathrm{NaCl}$. T: $\mathrm{T}_{2}$ generation of transgenic tobacco plants, WT: wild type (non-transgenic tobacco plants)

lower leaves turning yellow but upper leaves remaining green (Fig. 8b-c).

\section{Chlorophyll and Malondialdehyde (MDA) contents of transgenic plants after treatment with salt stress}

Leaf senescence is associated with chlorophyll breakdown and reactive oxygen species (ROS) accumulation $[19,20]$.
ROS-generated lipid peroxidation (reflected by MDA content) is an inherent feature of senescing cells and a source of ROS [21, 22]. To further analyze the effect of CaPAO overexpression on salt-induced leaf senescence in tobacco, chlorophyll and MDA contents were measured after salt treatment for 7 and 14 days, respectively (Fig. 8d-e). Before the salt stress, there were few obvious differences in

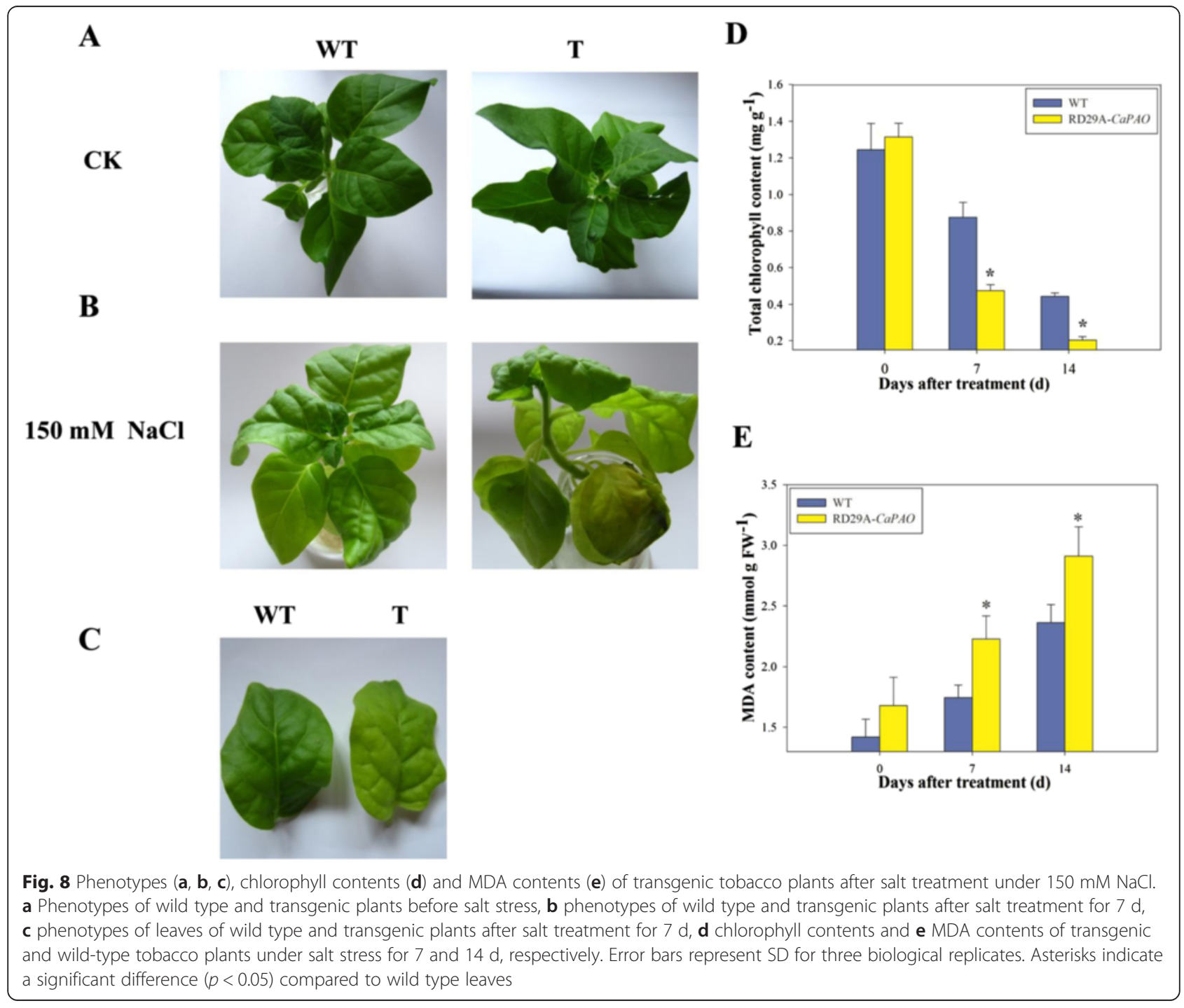


chlorophyll and MDA content between WT and transgenic leaves. Chlorophyll content of WT and transgenic leaves decreased by 0.37 and $0.84 \mathrm{mg} \cdot \mathrm{g}^{-1}$ after 7 days, and decreased by 0.44 and $0.20 \mathrm{mg} \cdot \mathrm{g}^{-1}$ after 14 days of salt treatment, respectively. The rate of chlorophyll breakdown in WT plants was higher than that in the transgenic plants. Correspondingly, MDA content gradually increased with elongated treated time. MDA content of WT and transgenic leaves increased by 0.32 and $0.55 \mathrm{mmol} \cdot \mathrm{g}^{-1}$ after 7 days, and increased by 2.36 and $2.91 \mathrm{mmol} \cdot \mathrm{g}^{-1}$ after 14 days of salt treatment, respectively. MDA content in WT plants was significantly lower than that in transgenic plants. These results indicated that overexpression of CaPAO could accelerate the chlorophyll breakdown and lipid peroxidation accumulation of transgenic tobacco plants under salt treatment. All these observations suggested that overexpression of $\mathrm{CaPAO}$ could accelerate saltinduced leaf senescence in tobacco.

\section{Discussion}

PAO opens the porphyrin macrocycle of pheophorbide $a$ and finally forms FCCs [3]. Here, we identified the $\mathrm{CaPAO}$ gene from pepper. $\mathrm{CaPAO}$ contained conserved domains that existed in PAO homologues. Furthermore, $\mathrm{CaPAO}$ shared high homology to other PAO proteins, especially SILLS1 and NtPAO. Bioinformatics analyses demonstrated that CaPAO should function as a PAO.

Tissue-specific analysis showed that $C a P A O$ was expressed in all tissues, and the expression level in leaves was higher than other tissues. The findings were similar to the previous results in maize and rice $[8,23]$, it was reported that expression of the OsPAO gene was detected in all tissues and at the highest level in leaves. However, the present results were different than those in A. thaliana [12], in which AtPAO transcript was higher in flowers and siliques than other tissues. These results revealed that $P A O$ is important to growth and development of all tissues, although its function may be distinct in different species.

Various biotic and abiotic stresses can affect growth and development of pepper plants, causing chlorophyll breakdown, cell death and finally premature senescence $[1,6,24,25]$. All of these consequences would impact the yield and quality of crop plants. PAO is an important intermediate in chlorophyll degradation, the expression and activity of which can be affected by various stresses. Wang et al. (2012) reported that relative expression of and $P A O$ in drought-stressed leaves was greatly upregulated in apple [26]. Microarray analysis indicated that upregulation of PAO in response to various stress conditions, coinciding with breakdown of Chlorophyll under these conditions [3, 27]. Results of the current study showed that transcript levels of the CaPAO gene were upregulated in pepper plants after treatment with various stresses, including high salinity, osmosis and Phytophthora capsici infection. These results suggested that CaPAO may be involved defense response to salt and osmotic stresses as well as Phytophthora capsici in pepper plants.

Previous studies have shown that phytohormone signaling pathways played an important role in mediating developmental processes and environmental responses in plants $[2,28]$. These hormones could cause the expression of stress-related genes, which in turn affect senescence progress of leaf. Many studies have proved that CaPAO gene expression or activity was regulated by hormones [29-32]. For instance, ABA can enhance the expression of $P A O$ in rice leaves [29]. Rodoni et al. (1998) suggested that the chlorophyll breakdown related to PAO activity in barley detached leaves, a rapid loss of chlorophyll in conjunction with an increase of PAO activity was occurred after treatment with $\mathrm{ABA}$ and MeJA [30]. In the current study, treatment with $\mathrm{ABA}, \mathrm{MeJA}$ or SA was found to increase the expression level of CaPAO. These findings suggested that the CaPAO gene may be involved in ABA-, JA- and SA-dependent signaling pathways. These results were different from those of a study in wheat reporting that $\mathrm{ABA}$ and MeJA enhanced the expression of TaPAO, while SA treatment did not cause significant changes in the level of this gene [6]. SA is the hormone involved in pathogen response and abiotic stress tolerance in pepper plant [22, 33]. In perennials, SA may be involved in the regulation of drought-induced leaf senescence as SA accumulation preceded chlorophyll breakdown and nitrogen mobilization [34]. Here, high salinity, osmosis and Phytophthora capsici infection induced CaPAO expression in pepper leaves. It is likely that salt, mannitol and Phytophthora capsici-induced SA accumulation may trigger $\mathrm{CaPAO}$ transcripts in pepper plants.

The function of the $P A O$ gene has been studied in many plants [8, 10, 35]. Tang et al. (2011) reported that chlorophyll breakdown was delayed compared to controls in leaves detached from leaves of PAO-silenced rice plants during dark-induced senescence [8]. Moreover, leaves with overexpression of PAO in Arabidopsis had lower chlorophyll content and earlier leaf yellowing than the control $4 \mathrm{~d}$ after dark treatment [35]. Leaf senescence was involved in chlorophyll degradation and accumulation of ROS. Drought-induced senescence in the leaves of apple was reflected in chlorophyll loss and an increase in the levels of ROS, with the induction of the $P A O$ gene [26]. In this research, the delay of chlorophyll degradation was detectable in the detached leaves from $\mathrm{CaPAO}$ silencing pepper plants 3 days after treatment with salt. Conversely, overexpression of CaPAO, induced by the stress-inducible promoter RD29A, could accelerate the rate of chlorophyll breakdown and lipid peroxidation of the transgenic plants under salt stress. The results of Tang et al. (2013) have shown that overexpressing TaPAO caused an accumulation of RCCs in 
wheat leaves [36]. RCCs have been proved to be phototoxic and caused cell deach, resulting in senescence [8,37]. All of these results demonstrated that $\mathrm{CaCP}$ played crucial role in pepper plant defense response to salt stresses, thus delaying leaf senescence.

\section{Conclusions}

Overall, we conclude that $\mathrm{CaPAO}$ plays an important role in senescence and chlorophyll degradation, as well as defense responses to various stresses. CaPAO was induced in natural senescence and various stresses. Silence of CaPAO resulted in a stay-green phenotype in pepper plants, and overexpression of $\mathrm{CaPAO}$ accelerated the process of salt-induced leaf senescence. In the future, the transgenic plants about overexpression or knockdown of $\mathrm{CaPAO}$ gene in pepper would be used for studying detail function of $C a P A O$.

\section{Methods}

Plant material

The B12 pepper cultivar was used for cloning and characterization of the CaPAO gene in this study. This early maturing variety was selected by a pepper research group in the College of Horticulture, Northwest A\&F University, China. The seeds were treated with warm water $\left(55^{\circ} \mathrm{C}\right)$ for $20 \mathrm{~min}$ and then incubated at $28{ }^{\circ} \mathrm{C}$ to accelerate germination under dark conditions. The seeds were rinsed twice every day until budding. The germinated seeds were sown in pots containing compost. Seedlings were placed in a growth chamber for a 16-h light/8-h dark cycle at $25^{\circ} \mathrm{C} / 21^{\circ} \mathrm{C}$, respectively.

\section{Cloning and sequence analysis of CaPAO gene}

To clone the CaPAO gene, total RNA was extracted from green mature leaves using the Trizol (Invitrogen) method, and first-strand cDNA synthesis was performed using Smart RACE cDNA amplification kit (Clontech). The reported nucleotide sequence of Nicotiana tabacum PAO (accession number: EU294211.1) was used as the query probe to retrieve homologous expressed sequence tag (EST) sequences of pepper in GenBank using the BLASTN protocol. Four overlapping pepper ESTs (accession numbers: GD077816, GD078012, GD072064 and GD077857) were chosen to be assembled into a splicing fragment without a complete ORF. To confirm the authenticity of the assembled pepper sequence, the ESTPAOF/EST-PAOR primer pair was used (Table 1). The design of both primers was based on the splicing sequence. The PCR reaction conditions were as follows: $94{ }^{\circ} \mathrm{C}$ for $5 \mathrm{~min} ; 35$ cycles at $94{ }^{\circ} \mathrm{C}$ for $30 \mathrm{~s}, 6{ }^{\circ} \mathrm{C}$ for $30 \mathrm{~s}, 72{ }^{\circ} \mathrm{C}$ for $1.5 \mathrm{~min}$; and finally an extension at $72{ }^{\circ} \mathrm{C}$ for $10 \mathrm{~min}$. The product was obtained, subcloned into a pMD19-T vector (TaKaRa) and sequenced.
Table 1 Primer sequences in this study

\begin{tabular}{|c|c|}
\hline primer & Sequence $\left(5^{\prime}-3^{\prime}\right)$ \\
\hline \multicolumn{2}{|l|}{ Cloning of CaPAO gene } \\
\hline EST-CaPAOF & СТTCACTCCCAATCACCAAACC \\
\hline EST-CaPAOR & TCGCTAACGAGCCCAAGATAAT \\
\hline 5'RACE-CAPAOGSP & CGGAGACTGAAACGCTITAGCTTC \\
\hline 5'RACE-CaPAONGSP & GAACAACCCACTGAGACCCAGATTT \\
\hline 3'RACE-CAPAOGSP & TTGGTGGCAGGTTITTCCGAGAT \\
\hline 3'RACE-CaPAONGSP & CGGCAGCACAGACAACCAACCAC \\
\hline \multicolumn{2}{|c|}{ quantitative real-time $P C R$} \\
\hline RT-CaPAOF & CAAGTTTGCCTACACCATTTCAG \\
\hline RT-CaPAOR & GCCCATITTCATCTAATCTCCCT \\
\hline CaUBI3F & TGTCCATCTGCTCTCTGTTG \\
\hline CaUBI3R & CACCCCAAGCACAATAAGAC \\
\hline \multicolumn{2}{|c|}{ Virus-induced gene silencing (VIGS) vector construction } \\
\hline TRV-CaPAOF & CAATGGCACCAGGAAAGACC \\
\hline TRV-CaPAOR & GCGTTGGACAAGACGGTAG \\
\hline CaPDSF & TGTTGTCAAAACTCCAAGGTCTGTA \\
\hline CAPDSR & TTTCTCCCACTTGGTTCACTCTTGT \\
\hline \multicolumn{2}{|c|}{ Tabacco genetic transformation } \\
\hline 2307-CaPAOF & ATGGCTTCTTCTTCTTCTTGTAT \\
\hline 2307-CaPAOR & TTCTAAATTTCCCTAGACTTCTAGTC \\
\hline RD29A-F & GAATAAATATCATACCGACATCA \\
\hline CAPAO-R & CAGTACTAGTAGATGATTCTTGTTC \\
\hline NtUBIF & TCCAGAAAGAGTCAACCCG \\
\hline NtUBIR & GACCTCAGTAGACAAAGCACAT \\
\hline
\end{tabular}

To isolate a full-length cDNA of the putative CaPAO gene with complete 5' and 3' regions, the RACE method was used. Gene-specific primers (5' -GSP, $5^{\prime}$-NGSP, 3'-GSP, and $3^{\prime}$-NGSP) were designed according to the partial cDNA sequence (Table 1). The 5'-GSP (external) and 5'NGSP (internal) primers were used to isolate 5 '-end sequences, while the 3'-GSP (external) and 3'-NGSP (internal) primers were used for isolation of $3^{\prime}$-end sequences. Nested PCR was performed in the 5'RACE and 3'RACE procedures. The amplified PCR products were ligated into the pMD19-T vector and sequenced. Finally, all acquired sequences were assembled into a single sequence with a complete ORF using the Contig Expression software and BLAST online software (http://www.ncbi.nlm.gov/blast).

The putative $\mathrm{CaPAO}$ cDNA and protein sequences were analyzed by the DNAMAN software 5.2.2 and BLAST online software. Prediction of the subcellular localization of the putative signal peptide, chloroplast transit peptides and its cleavage site were carried out using the CBS prediction server's online program (http://www.cbs.dtu.dk/services/). The $\mathrm{p} I$ and MW of the putative protein were analyzed with the $\mathrm{p} / \mathrm{MW}$ program (http://www.expasy.org/), and its secondary structure was predicted using the Scratch Protein 
Predictor online program (http://scratch.proteomics.ics. uci.edu). The multiple sequence alignments of $\mathrm{CaPAO}$ and other PAO proteins were performed with DNAMAN software. The phylogenetic tree was constructed using the MEGA5.05 program with the neighbor-joining method.

\section{CaPAO Gene expression pattern analysis Tissue-specific expression of CaPAO gene}

To evaluate the CaPAO gene expression levels in different tissues, root, stem, leaf and flower samples were collected from the untreated B12 pepper cultivar. To examine CaPAO expression pattern during leaf development, leaves were harvested at young, fully expanded and senescent stage, respectively. All samples were frozen in liquid nitrogen and kept at $-80{ }^{\circ} \mathrm{C}$ prior to RNA extraction.

\section{Stress treatments}

Signaling molecules as well as abiotic and biotic stresses were used to treat six-leaf stage pepper plants. For treatments with signaling molecules, plant hormones were applied by spraying pepper plant leaves with either $0.57 \mathrm{mM}$ ABA, $5 \mathrm{mM}$ SA or $1 \mathrm{M}$ MeJA dissolved in $0.05 \%(\mathrm{v} / \mathrm{v})$ ethanol. The control plants were treated with distilled water containing $0.05 \%(\mathrm{v} / \mathrm{v})$ ethanol. After $0,2,4,8,12$, 24 and $48 \mathrm{~h}$ of treatment, pepper leaves were sampled. For salt and drought stress treatments, the pepper plants were uprooted from the soil, and their roots were soaked in $400 \mathrm{mM} \mathrm{NaCl}$ and $400 \mathrm{mM}$ mannitol, respectively. The control seedlings were treated with sterile water. After 0 , $2,4,8,12$ and $24 \mathrm{~h}$ of treatment, pepper leaves were collected. For the fungal pathogen treatment, pepper plants were inoculated with Phytophthora capsici (HX-9 strain) by the root-drenching method, while control plants were treated with sterile distilled water [38]. The inoculated and control plants were incubated in a growth chamber at $28{ }^{\circ} \mathrm{C}$ under a 16-h light/8-h dark photoperiod cycle with $60 \%$ relative humidity. The samples were collected at 0,3 , $6,12,24,48,72$ and $96 \mathrm{~h}$ intervals. All samples were frozen immediately in liquid nitrogen and kept at $-80{ }^{\circ} \mathrm{C}$ prior to RNA extraction.

\section{Isolation RNA and genomic DNA}

Total RNA was extracted from pepper and tobacco using the Trizol (Invitrogen) method. Contaminated genomic DNA was digested by RNase-free DNase I (Promega). The concentration and purity of total RNA were determined spectrophotometrically using a NanoDrop instrument (Thermo Scientific NanoDrop 2000C Technologies). The first-strand cDNA was synthesized according to the instructions of the PrimeScript ${ }^{\mathrm{ma}}$ Kit (TaKaRa). Genomic DNA was extracted from tobacco mature leaves using CTAB method.

\section{Quantitative real-time PCR and semi-quantitative RT-PCR analysis}

In order to identify the CaPAO expression patterns in different pepper tissues without treatment and in leaves under various stresses, qRT-PCR was performed using SYBR $^{\circ}$ Premix Ex Taq $^{\text {tm }}$ II (TaKaRa) in $20 \mu$ reaction volume containing 10.0 $\mu \mathrm{l} \mathrm{S_{BR } { } ^ { \circ } \text { Premix Ex Taq }}{ }^{\text {Tw }}$ II, $2.0 \mu \mathrm{l}$ diluted cDNA, and $0.8 \mu \mathrm{l}$ of forward and reverse primers. The amplification was completed with the following cycling parameters: $95^{\circ} \mathrm{C}$ for $1 \mathrm{~min}$, followed by 40 cycles of $95{ }^{\circ} \mathrm{C}$ for $10 \mathrm{~s}, 57{ }^{\circ} \mathrm{C}$ for $20 \mathrm{~s}$ and $72{ }^{\circ} \mathrm{C}$ for $20 \mathrm{~s}$. The CaUBI3 gene (accession number: AY486137.1) was used as an internal control (reference gene) in this study (Table 1). The relative gene expression levels were calculated using the $2^{-\Delta \Delta \mathrm{Ct}}$ comparative threshold method [39]. All samples were performed in triplicate, and each had at least three independent biological replicates.

Semi-quantitative RT-PCR was performed for analysis the expression level of $C a P A O$ gene in transgenic tobacco, and the RD29A-F and CaPAO-R primer pair were used. The PCR cycles were $1 \mathrm{~min}$ at $95^{\circ} \mathrm{C}$ followed by 29 cycles at $30 \mathrm{~s}$ at $95^{\circ} \mathrm{C}, 30 \mathrm{~s}$ at $53^{\circ} \mathrm{C}$, and $60 \mathrm{~s}$ at $72{ }^{\circ} \mathrm{C}$ followed by an extension for $10 \mathrm{~min}$ at $72{ }^{\circ} \mathrm{C}$. The PCR products were separated on $2 \%$ agarose gels stained with ethidium bromide to detect expression degree of $C a P A O$ gene. The $N t U B I$ gene was used as a reference gene.

\section{Plasmid construction and inoculation of VIGS agrobacterium in pepper plants Construction of VIGS Plasmids TRV2-CaPAO and TRV2-CaPDS} A CaPAO sequence fragment was amplified using genespecific primers with restriction sites $\mathrm{XbaI}$ (forward) and $B a m H I$ (reverse) and inserted into the pTRV2 vector to generate TRV2-CaPAO. The primers for VIGS are shown in Table 1. The CaPDS gene (phytoene desaturase from $C$. annuum, accession number: X68058.1) was used for determining the effectiveness of VIGS in this study, and the TRV2-CaPDS vector was designed by our laboratory. Finally, these plasmids (pTRV1, pTRV2, TRV2-CaPDS and TRV2-CaPAO) were each transformed into the Agrobacterium tumefaciens strain GV3101.

\section{Inoculation of VIGS Agrobacterium in pepper plants}

Preparation of $A$. tumefaciens harboring pTRV1, pTRV2, TRV2-CAPDS or TRV2-CaPAO vectors were described by Liu et al. [40]. Before inoculation, cultures containing pTRV1 and pTRV2 or their derivatives (TRV2-CaPDS or TRV2-CaPAO) were mixed at a 1:1 ratio. The mixtures of Agrobacterium were inoculated into the fully-expanded cotyledons of the B12 pepper cultivars. Plants were placed in a growth chamber at $18{ }^{\circ} \mathrm{C}$ and $60 \%$ relative humidity for $2 \mathrm{~d}$. Subsequently, the plants were grown at $23{ }^{\circ} \mathrm{C}$ under a 16-h light/8-h dark photoperiod cycle and $60 \%$ 
relative humidity. At 45 days after inoculation, the leaves were used for characterization and analysis of CaPAO.

\section{Treatment of CaPAO-silenced plants}

To analyse salt stress of the gene-silenced plants, the leaf discs from empty vector control-treated (TRV2:00) and CaPAO-silenced (TRV2: CaPAO) plants were floated in various concentrations $(0,300,400$ and $500 \mathrm{mM})$ of $\mathrm{NaCl}$ solution with continuous lighting at $25{ }^{\circ} \mathrm{C}$ for 3 days. The symptoms of leaf discs were observed during treatment, and the chlorophyll content was determined after $3 \mathrm{~d}$.

\section{Tobacco genetic transformation of the CaPAO gene Construction of transgenic vector PVBG2307-RD29A-CaPAO}

The $C a C P$ sequence with a complete ORF was amplified using a primer pair 2307-CaPAOF/2307-CaPAOR and inserted into PVBG2307 plasmid possessing BamHI and KpnI restriction enzyme sites to yield PVBG2307-CaPAO. Then the $R D 29 A$ promoter sequence from RD29A-T vector was cloned into PVBG2307-CaPAO vector using digestion of HindIII and XbaI restriction enzymes. PVBG2307PRD29A-CaPAO vector was constructed successfully. Finally, the plasmid was transformed into the Agrobacterium tumefaciens strain GV3101.

\section{Plant transformation and selection of $T_{2}$ generation of transgenic tobacco plants}

Tobacco plants (Nicotiana tabacum cv. Bairihong) were used for all subsequent analyses, the successful transformed plants with leaf disc method were selected and incubated as previously described by Li et al. [41]. The primers RD29A-F and CaPAO-R were used to conform the transgenic tobacco seedlings at genomic DNA level, and a second pair of primers, 2307-CaPAOF and CaPAO-R were used to further confirm the transgenic status of transgenic tobacco plants. Growth conditions of tobacco seedlings and seed selection were followed according to the method of previous research [42]. The $\mathrm{T}_{2}$ offspring of transgenic tobacco plants were used for all subsequent experiments.

\section{Treatment of transgenic tobacco plants}

Seedlings of WT and one tobacco line $(\mathrm{T})$ at the stage of four true leaves were carefully removed from the pots and their roots washed with tap water prior to solution culture in $1 / 4$ strength Hoagland's solution. After seven to eight true leaves stage, the seedlings were cultured with $1 / 2$ Hoagland's solution supplied with $150 \mathrm{mM} \mathrm{NaCl}$ solution. Leaves were harvested at 0,7 , and $14 \mathrm{~d}$ after salt treatment, and total chlorophyll and MDA contents were measured. All treatments were repeated three times and arranged in a randomized complete block design.
Determination of chlorophyll content and MDA content For determination of chlorophyll content, leaf samples $(0.5 \mathrm{~g})$ were grinded into a fine powder in the presence of liquid nitrogen, mixed with $8 \mathrm{ml}$ acetone $80 \%(\mathrm{v} / \mathrm{v})$ and kept overnight at $4{ }^{\circ} \mathrm{C}$ to extract chlorophyll. The supernatant containing chlorophyll was obtained after centrifugation at $10,000 \times g$ for $10 \mathrm{~min}$ at $4{ }^{\circ} \mathrm{C}$. The chlorophyll content was measured by a spectrophotometric method, and results were expressed in milligrams of total chlorophyll per gram fresh weight of tissues [43]. The MDA content of treated leaves was measured according to the method of Buege and Aust [44]. All experiments were replicated three times.

\section{Primers used in this study}

Primers used for cloning, quantitative real-time PCR analysis, semi-quantitative RT-PCR Analysis, VIGS and transgenosis are listed in Table 1.

\section{Statistical analysis}

All data are presented as the mean \pm standard deviation (SD) of three replicates. Quantitative data were analyzed using Statistical Analysis System software (SAS Institute, version 8.2) following one-way analysis of variance (ANOVA). Differences among means were analyzed at a significance level of 0.05 .

\begin{abstract}
Abbreviations
ABA: Abscisic acid; bp: base pair(s); DREs: Dehydration- responsive elements; EST: Expressed sequence tag; FCC: Fluorescent chlorophyll catabolite; MDA: Malondialdehyde; MeJA: Methyl jasmonate; MW: Molecular weight; $\mathrm{NaCl}$ : sodium chloride; ORF: Open reading frame; PAO: Pheophorbide a oxygenase; pheide $a$ : pheophorbide $a$; pl: isoelectric point; qRT-PCR: quantitative real-time PCR; RACE: Rapid amplification of CDNA ends; SA: Salicylic acid; TRV: Tobacco rattle virus; UTR: Untranslated region; VIGS: Virus-induced gene silencing; WT: Wild- type.
\end{abstract}

\section{Competing interests}

The authors declare that they have no competing interests.

\section{Authors' contributions}

Conceived and designed the experiments: H-JX, D-WL, Z-HG. Performed the experiments: H-JX, L-KK, W-GC. Analyzed the data: H-JX, L-KK, D-WL, W-GC. Contributed reagents/materials/analysis tools: Z-HG. Wrote the paper: H-JX, $\mathrm{M}-\mathrm{HA}$. All authors read and approved the final manuscript.

\section{Acknowledgments}

This research was supported by the National Natural Science Foundation of China (No. 31272163) the Shaanxi Provincial Science and Technology Coordinating Innovative Engineering Project (2012KTCL02-09), and the Hangzhou Municipal Science and Technology Commission Project (20140932H01).

\section{Author details}

${ }^{1}$ College of Horticulture, Northwest A\&F University, Yangling, Shaanxi, P. R. China. ${ }^{2}$ College of Horticulture, Henan Agricultural University, Zhengzhou, Henan, P. R. China. ${ }^{3}$ Faculty of Agriculture, Zagazig University, Zagazig, Sharkia P. R. Egypt. ${ }^{4}$ Institute of Vegetables, Hangzhou Academy of Agricultural Sciences, Hangzhou, Zhejiang, P. R. China.

Received: 22 April 2015 Accepted: 6 October 2015

Published online: 24 October 2015 


\section{References}

1. Guo Y, Gan SS. Convergence and divergence in gene expression profiles induced by leaf senescence and 27 senescence-promoting hormonal, pathological and environmental stress treatments. Plant Cell Environ. 2012;35(3):644-55.

2. Lim PO, Kim HJ, Nam HG. Leaf senescence. Annu Rev Plant Biol. 2007;58:115-36.

3. Hörtensteiner S. Chlorophyll degradation during senescence. Annu Rev Plant Biol. 2006:57:55-77.

4. Hörtensteiner S, Kräutler B. Chlorophyll breakdown in higher plants. Biochimica Et Biophysica Acta-Bioenergetics. 2011;1807(8):977-88.

5. Li P, Ma Y, Li X, Zhang L, Wang Y, Wang N. Cloning and expressional characterization of soybean GmL/s1 gene. Chin Sci Bull. 2006;51:1210-8.

6. Ma N, Ma X, Li A, Cao X, Kong L. Cloning and expression analysis of wheat pheophorbide a oxygenase gene TaPaO. Plant Mol Biol Rep. 2012;30(5):1237-45.

7. Spassieva S, Hille J. A lesion mimic phenotype in tomato obtained by isolating and silencing an L/s1 homologue. Plant Sci. 2002;162(4):543-9.

8. Tang Y, Li M, Chen Y, Wu P, Wu G, Jiang H. Knockdown of OsPAO and OsRCCR1 cause different plant death phenotypes in rice. J Plant Physiol. 2011;168(16):1952-9.

9. Chung DW, Pružinská A, Hörtensteiner S, Ort DR. The role of pheophorbide a oxygenase expression and activity in the canola green seed problem. Plant Physiol. 2006;142(1):88-97.

10. Gray J, Close PS, Briggs SP, Johal GS. A novel suppressor of cell death in plants encoded by the L/s1 gene of maize. Cell. 1997;89(1):25-31.

11. Gray J, Janick-Buckner D, Buckner B, Close PS, Johal GS. Light-dependent death of maize I/s1 cells is mediated by mature chloroplasts. Plant Physiol. 2002;130(4):1894-907.

12. Prǔzinská A, Tanner G, Aubry S, Anders I, Moser S, Müller T, et al. Chlorophyll breakdown in senescent Arabidopsis leaves. Characterization of chlorophyll catabolites and of chlorophyll catabolic enzymes involved in the degreening reaction. Plant Physiol. 2005;139(1):52-63.

13. Prǔzinská A, Tanner G, Anders I, Roca M, Hörtensteiner S. Chlorophyll breakdown: Pheophorbide a oxygenase is a Rieske-type iron-sulfur protein, encoded by the accelerated cell death 1 gene. Proc Natl Acad Sci U S A. 2003;100(25):15259-64.

14. Tanaka R, Hirashima M, Satoh S, Tanaka A. The Arabidopsis-accelerated cell death gene ACD1 is involved in oxygenation of pheophorbide $a$ : inhibition of the pheophorbide a oxygenase activity does not lead to the "Stay-Green" phenotype in Arabidopsis. Plant Cell Physiol. 2003;44(12):1266-74.

15. Hirashima M, Tanaka $R$, Tanaka A. Light-independent cell death induced by accumulation of pheophorbide $a$ in Arabidopsis thaliana. Plant Cell Physiol. 2009;50(4):719-29.

16. Qiu WM, Liu MY, Qiao GR, Jiang J, Xie LH, Zhuo RY. An isopentyl transferase gene driven by the stress-inducible rd29A promoter improves salinity stress tolerance in transgenic tobacco. Plant Mol Biol Rep. 2012;30(3):519-28.

17. Li F, Han YY, Feng YA, Xing SC, Zhao MR, Chen YH, et al. Expression of wheat expansin driven by the RD29 promoter in tobacco confers water-stress tolerance without impacting growth and development. J Biotechnol. 2013;163(3):281-91.

18. Yamaguchi-Shinozaki K, Shinozaki K. A novel cis-acting element in an Arabidopsis gene is involved in responsiveness to drought, lowtemperature, or high-salt stress. Plant Cell. 1994;6(2):251-64.

19. Rosenvasser $\mathrm{S}$, Mayak S, Friedman $\mathrm{H}$. Increase in reactive oxygen species (ROS) and in senescence-associated gene transcript (SAG) levels during dark-induced senescence of Pelargonium cuttings, and the effect of gibberellic acid. Plant Sci. 2006;170(4):873-9.

20. Vanacker H, Sandalio LM, Jimenez A, Palma JM, Corpas FJ, Meseguer V, et al. Role for redox regulation in leaf senescence of pea plants grown in different sources of nitrogen nutrition. J Exp Bot. 2006;57(8):1735-45.

21. Chopra KR. Leaf senescence and abiotic stresses share reactive oxygen species-mediated chloroplast degradation. Protoplasma. 2012;249(3):469-81.

22. Xiao HJ, Yin YX, Chai WG, Gong ZH. Silencing of the CaCP gene delays saltand osmotic- induced leaf senescence in Capsicum annuum L. Int J Mol Sci. 2014;15(5):8316-34.

23. Yang M, Wardzala E, Johal GS, Gray J. The wound-inducible LIsI gene from maize is an orthologue of the Arabidopsis Acd1 gene, and the LLS1 protein is present in non-photosynthetic tissues. Plant Mol Biol. 2004;54(2):175-91.

24. Mur LAJ, Aubry S, Mondhe M, Kingston-Smith A, Gallagher J, Timms-Taravella E, et al. Accumulation of chlorophyll catabolites photosensitizes the hypersensitive response elicited by Pseudomonas syringae in Arabidopsis. New Phytol. 2010;188(1):161-74

25. Roca M, Mínguez-Mosquera Ml. Chlorophyll catabolism pathway in fruits of Capsicum annuum (L.): stay-green versus red fruits. J Agric Food Chem. 2006:54(11):4035-40.

26. Wang $P$, Yin LH, Liang D, Li C, Ma FW, Yue ZY. Long-term exogenous application of melatonin delays drought-induced leaf senescence in apple. J Pineal Res. 2013;54(3):292-302.

27. Thomas H, Ougham H, Hörtensteiner S. Recent advances in the cell biology of chlorophyll catabolism. Adv Bot Res. 2001;35(1):1-52.

28. Rajendra B, Jones JD. Role of plant hormones in plant defence responses. Plant Mol Biol. 2009;69(4):473-88.

29. Jiang $H$, Li M, Liang $N$, Yan $H$, Wei $Y$, Xu X, et al. Molecular cloning and function analysis of the stay green gene in rice. Plant J. 2007;52(2):197-209.

30. Rodoni S, Schellenberg M, Matile P. Chlorophyll breakdown in senescing barley leaves as correlated with phaeophorbidea oxygenase activity. J Plant Physiol. 1998;152:139-44.

31. Shan X, Wang J, Chua L, Jiang D, Peng W, Xie D. The role of Arabidopsis rubisco activase in jasmonate-induced leaf senescence. Plant Physiol. 2011;155(2):751-64.

32. Zhang X, Zhang Z, Li J, Wu L, Guo J, Ouyang L, et al. Correlation of leaf senescence and gene expression/activities of chlorophyll degradation enzymes in harvested Chinese flowering cabbage (Brassica rapa var. parachinensis). J Plant Physiol. 2011;168(17):2081-7.

33. Wang JE, Liu KK, Li DW, Zhang YL, Zhao Q, He YM, et al. A novel peroxidase CanPOD gene of pepper is involved in defense responses to Phytophtora capsici infection as well as abiotic stress tolerance. Int J Mol Sci. 2013;14(2):3158-77.

34. Abreu ME, Munné-Bosch S. Salicylic acid may be involved in the regulation of drought-induced leaf senescence in perennials: A case study in field-grown Salvia officinalis L. Plants. Environ Exp Bot. 2008;64(2):105-12

35. Sakuraba Y, Schelbert S, Park SY, Han SH, Lee BD, Andres CB, et al. STAYGREEN and chlorophyll catabolic enzymes interact at light-harvesting complex II for chlorophyll detoxification during leaf senescence in Arabidopsis. Plant Cell. 2012;24(2):507-18

36. Tang CL, Wang XJ, Duan XY, Wang XD, Huang LL, Kang ZS. Functions of the lethal leaf-spot 1 gene in wheat cell death and disease tolerance to Puccinia striiformis. J Exp Bot. 2013;64(10):2955-69.

37. Prǔzinská A, Anders I, Aubry S, Schenk N, Tapernoux-Luthi E, Muller T, et al. In vivo participation of red chlorophyll catabolite reductase in chlorophyll breakdown. Plant Cell. 2007;19(1):369-87.

38. Wang JE, Li DW, Zhang YL, Zhao Q, He YM, Gong ZH. Defence responses of pepper (Capsicum annuum L.) infected with incompatible and compatible strains of Phytophthora capsici. Eur J Plant Pathol. 2013;136(3):625-38.

39. Livak KJ, Schmittgen TD. Analysis of relative gene expression data using realtime quantitative PCR and the $2_{T}^{-\Delta \Delta C}$ method. Methods. 2001;25(4):402-8.

40. Liu Y, Schiff M, Dinesh-Kumar SP. Virus-induced gene silencing in tomato. Plant J. 2002;31(6):777-86.

41. Li Z, Wang S, Tao QY, Pan JS, Si LT, Gong ZH, et al. A putative positive feedback regulation mechanism in CSACS2 expression suggests a modified model for sex determination in cucumber (Cucumis sativus L.). J Exp Bot. 2012;63(12):4475-84.

42. Yin YX, Guo WL, Zhang YL, Ji JJ, Xiao HJ YF, et al. Cloning and characterisation of a pepper aquaporin, $C a A Q P$, which reduces chilling stress in transgenic tobacco plants. Plant Cell Tiss Organ Cult. 2014;118(3):431-44.

43. Inskeep WP, Bloom PR. Extinction coefficients of chlorophyll a and b in n, ndimethylformamide and $80 \%$ acetone. Plant Physiol. 1985;77(2):483-5.

44. Buege JA, Aust SD. Microsomal lipid peroxidation. Method Enzymol. 1978;52(52):302-10. 